\title{
Cosecha de agua de lluvia como tecnología de conservación de los manantiales amenazados, Chachapoyas
}

\section{Harvest of rainwater as a conservation technology for threatened springs, Chachapoyas}

\author{
Carmen Natividad Vigo Mestanza ${ }^{1 *}$ (D), Lily del Pilar Juárez Contreras ${ }^{1}$, Manuel Oliva ${ }^{1}$ (D)
}

\section{RESUMEN}

La afectación a los cuerpos de agua producto de las diferentes actividades humanas es un problema de índole mundial al que el Perú no es ajeno. En ese contexto, esta investigación buscó evaluar la eficiencia de la cosecha de agua de lluvia como una tecnología de conservación de los manantiales que vienen siendo amenazados en el anexo Pomacochas, distrito de Leimebamba, Chachapoyas. Para ello se usó el instrumento de la encuesta y se aplicó a 14 pobladores del anexo. Luego se midieron las dimensiones del techo de sus viviendas. Con la información recolectada se promedió cuanto era el porcentaje de familias que estaban dispuestas a implementar el sistema de cosecha de agua de lluvia. Se obtuvo que el $50 \%$ de la población estaría dispuesta a hacerlo, y este porcentaje se incrementaría si las personas recibieran información de esta tecnología. Además se pudo observar que la mayoría de los techos son de calamina, que es el material más apropiado por razones de salubridad y duración. Si se implementaría esta tecnología, se reduciría la presión sobre el recurso hídrico, en especial producto de las actividades domésticas.

Palabras claves: Manantial, ganadería, deforestación, sistema colecta, agua de lluvia.

\begin{abstract}
The affectation to the bodies of water product of the different human activities is a problem of world-wide nature, to which Peru is not foreign. In this context, this research sought to evaluate the efficiency of the rainwater harvesting as a conservation technology for the springs that are being threatened in the Pomacochas Annex, Leymebamba district, Chachapoyas. For this, the survey instrument was used. The survey was applied to 14 residents of the annex. Then the dimensions of the roof of their homes were measured. With the information collected, the percentage of families that were willing to implement the rainwater harvesting system was averaged. It was obtained that $50 \%$ of the population is willing to implement a rainwater collection system, this percentage would increase if people received information about this technology. In addition it was possible to observe that most of the ceilings are of calamine, which is the most appropriate material for reasons of healthiness and duration. If this technology were to be implemented, the pressure on the water resource would be reduced, especially as a result of domestic activities.
\end{abstract}

Keywords: Spring, livestock, deforestation, collection system, rainwater.

\footnotetext{
${ }^{1}$ Universidad Nacional Toribio Rodríguez de Mendoza de Amazonas (UNTRM-A), Instituto de Investigación para el Desarrollo Sustentable de Ceja de Selva, Calle Higos Urco N³42-350-356, Calle Universitaria N³04, Chachapoyas, Perú

"Autor de correspondencia. E-mail: cvigo@indes-ces.edu.pe
} 


\section{INTRODUCCIÓN}

La población a nivel mundial alcanzó los 7300 millones el año 2015, y su proyección para el futuro es alarmante: 9700 millones para el 2050 (ONU, 2015).

En el Perú la tasa de crecimiento es de 1,3\% el año 2016 (Banco Mundial, 2017), y en Chachapoyas la población dicho año alcanzó los 56100 habitantes (INEI, 2016). Se observa un rápido crecimiento de la misma, siendo este más notorio en las áreas urbanas. Así, en Perú por ejemplo el $78 \%$ de la población se encuentra asentada en el área urbana (INEI, 2007)

Este crecimiento voraz de la población asentada en las áreas urbanas genera una gran presión sobre los recursos naturales en general, ya que se hace necesaria una mayor cantidad de recursos para satisfacer las necesidades básicas de la población (UNESCO, 2017). Sin embargo, uno de los recursos más escasos y sobre el cual se ejerce una mayor presión en las áreas urbanas lo constituye el recurso hídrico, ya que este se utiliza en múltiples actividades como la actividad ganadera, agricultura, alimentación, y aseo, entre otras (Correa et al., 2011).

En el Perú se encuentran abundantes fuentes de agua dulce (ANA, 2013), que harían pensar que la presión sobre este recurso no es significativa. Sin embargo, estas no están distribuidas necesariamente donde se asienta la mayor parte de la población, ya que el $66 \%$ de la población peruana se encuentra ubicada en la vertiente del océnao Pacífico, que es la de menor disponibilidad hídrica (MINAGRI, 2012), agravando el problema de la sobreexplotación del recurso hídrico, debido a que las escasas fuentes de agua que se encuentran cercanas o dentro de las áreas urbanas son explotadas para el consumo directo, la realización de las actividades diarias, actividades agrícolas y ganaderas (UNESCO, 2003), incluso llegando a sobreexplotar en los meses de estiaje (Teodoro et al., 2013).

Una manera de evitar la sobreexplotación de los recursos hídricos es disponer de una fuente alternativa para utilizarla en la época de estiaje (Delgado et al., 2015). Una de las maneras más eficiente de obtenerla es mediante la cosecha del agua de lluvia (Abu y Tamini, 2011). Está práctica ha demostrado ser una tecnología eficiente para la protección de manantiales y fuentes de agua amenazados por el crecimiento poblacional, además de proporcionar una disminución de la temperatura de sensación cuando se emplean de manera conjunta con otras tecnologías como los jardines de azotea (An et al., 2015). Además es importante mencionar que esta tecnología no solo es positiva en términos ambientales sino en términos económicos ya que el ahorro en el consumo del agua se considera alrededor del 30\% (Rashidi et al., 2013). Por lo tanto si se consigue demostrar la eficiencia de la cosecha de agua de lluvia, en un futuro podremos hablar de personas adoptando esta importante tecnología en miras de la protección de los recursos hídricos (Ricardo et al., 2015).

Lo anteriormente descrito nos conduce a buscar la manera de evaluar la eficiencia de la cosecha de agua de lluvia en la protección de fuentes de agua amenazadas por el crecimiento poblacional en la ciudad de Chachapoyas.

\section{MATERIALES Y MÉTODOS}

\section{Ubicación del área de estudio}

El área de estudio se encuentra ubicada en el anexo Pomacochas, distrito de Leymebamba, provincia de Chachapoyas en el departamento de Amazonas (Figura 1).

\section{Metodología empleada}

El trabajo se inició con la revisión de información referida al proyecto de investigación. Luego se hizo una visita exploratoria al anexo para realizar el conteo de viviendas que se encuentran habitadas, además de identificar a productores que estén dispuestos a participar con el estudio durante la etapa de ejecución. También se calculó del tamaño muestral considerando que se garantice la representatividad.

Luego se procedió a diseñar la encuesta en función a la información que queríamos obtener con distintas fases de campo:

Fase de campo 


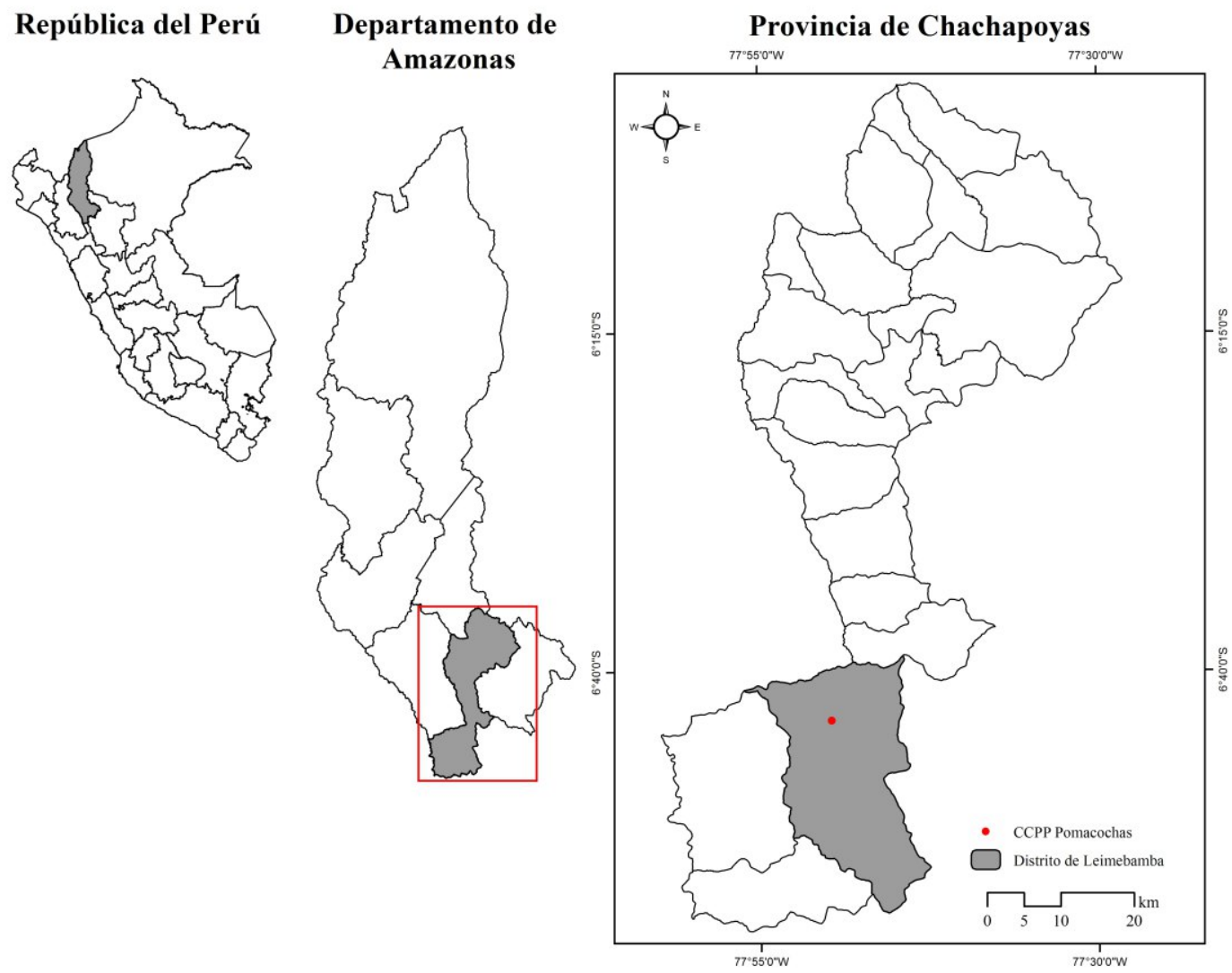

Figura 1. Ubicación del área de estudio en el Distrito de Leymebamba, provincia de Chachapoyas, Departamento Amazonas.

Se calcularon las dimensiones de los techos de las casa para conocer el área que poseen y conocer si es de un material apto para realizar la cosecha de agua de lluvia. Se aplicaron encuestas al número de pobladores del anexo de Pomacochas que nos arrojó el tamaño muestral.

\section{$\underline{\text { Visita y caracterización del manantial }}$}

Se realizaron visitas para determinar el estado situacional del manantial, y se registrará la existencia de focos de contaminación, la abundancia de la vegetación aledaña y su caudal y volumen aproximado.

\section{Diagnóstico de los reservorios de agua existentes}

Se visitaron los reservorios de la zona y se recolectó información respecto al volumen que tiene, a la limpieza que recibe entre otros aspectos relacionados a la calidad y disponibilidad del recurso hídrico a los mismos pobladores de la zona.

\section{Fase de gabinete}

Para la elaboración de los mapas de ubicación se hizo uso del software ArcGis v. 10.2. y Google Earth. Se realizaron los cálculos de las dimensiones de los techos de las viviendas y el volumen de agua que se puede captar a nivel doméstico y colectivo.

Además se hizo el procesamiento de las encuestas aplicadas. Se recolectó información respecto a las características físicas como ph, conductividad, turbidez y oxígeno disuelto; químicas como alcalinidad, dureza, cloruros, nitratos, nitritos, amonio, fosfatos, sulfatos, demanda química de oxígeno (DQO), demanda bioquímica de oxígeno (DBO); y microbiológicas de las muestras de agua de la zona de trabajos anteriores.

\section{Fuente de agua}

La fuente de agua abastece a un total de 14 familias del anexo de Pomacochas, ya sea de manera directa o a través del sistema de tuberías. La vegetación ribereña que acompaña a la fuente de agua está constituida básicamente por especies arbustivas como la chiska (Baccharis latifolia), aliso (Alnus acuminata), sauco (Sambucus peruviana), pajuro (Erythrina edulis) (Figura 2). 


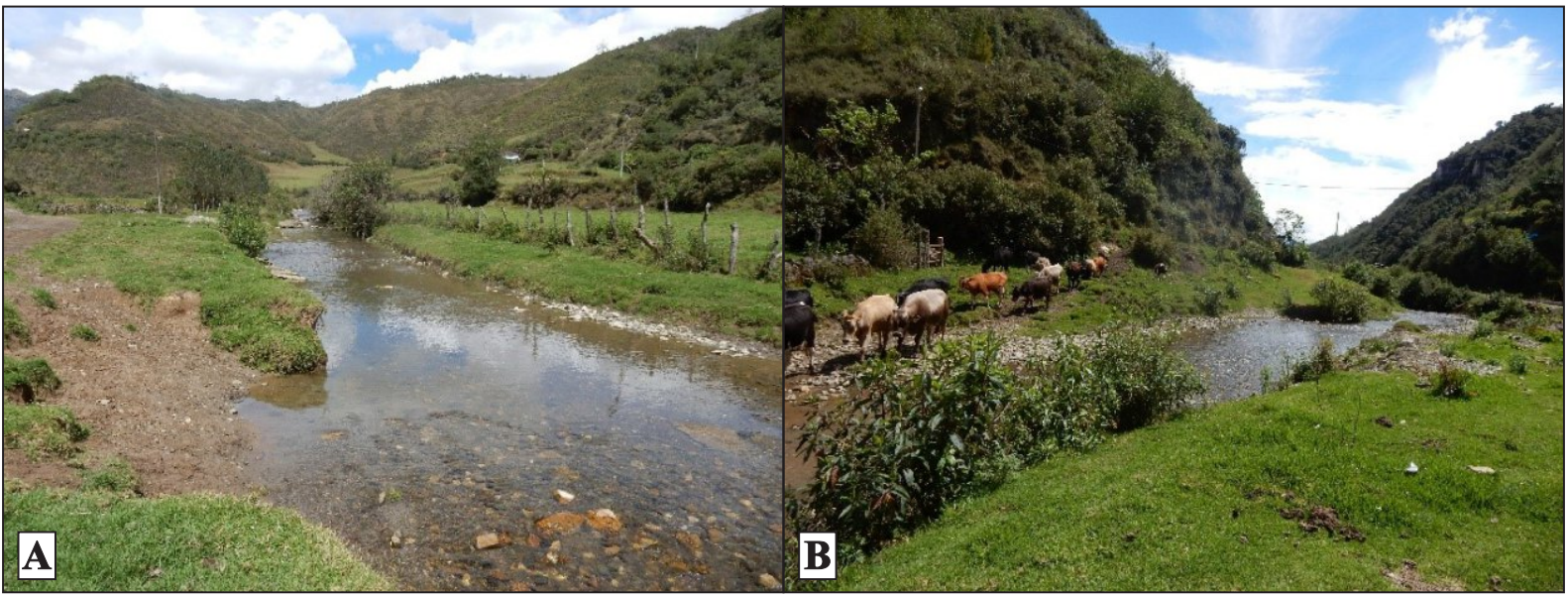

Figura 2. A, Fuente de agua. B, Vegetación ribereña.

\section{RESULTADOS}

\section{Resultados de la encuesta aplicada}

Se aplicó la encuesta a un total de 14 pobladores de la zona de estudio y se obtuvieron los siguientes resultados. El número promedio de habitantes por vivienda oscila entre 4 y $6(50,0 \%)$. La edad de los pobladores oscila entre los 41 y los 50 años (35,7\%). El género predominante fue el femenino con un $71,4 \%$. Tienen como ocupación principal la agricultura y ganadería (64,3\%). El nivel de estudios alcanzados es del nivel primario en su mayoría (50,0\%).

Con respecto al material de sus viviendas el material predominante es el adobe $(42,9 \%)$ y el $92,9 \%$ cuenta con servicio eléctrico. El combustible que usan para cocinar es la leña en su gran mayoría (64,3\%) seguido del gas en combinación de la leña $(28,6 \%)$.

Los techos de las viviendas son de calamina, esternil, teja y las combinaciones de las mismas. Sin embargo, la calamina es el material más utilizado $(71,4 \%)$. Con respecto a si tenían o no huerta familiar, el 57,1\% respondió que sí con dimensiones promedio alrededor de un cuarto de hectárea (62,5\%). Lo que más cultivan son verduras (62,5\%). Finalmente, el 64,3\% manifestó tener animales domésticos, con mayor predominancia gallinas $(33,3 \%)$.

El 85,7\% de la población no conoce cuál es su empresa prestadora del servicio de agua y un 14,3\% asegura que lo es la Municipalidad Distrital. El 71,4\% de la población cree que el agua que llega a su domicilio es abundante y de buena calidad todo el año, sin embargo el $50 \%$ no conoce la fuente de la que proviene el agua. Las amenazas principales que creen que enfrentan son la sequía $(7,1 \%)$ y la ganadería extensiva $(7,1 \%)$, mientras que el restante $85,7 \%$ cree que no enfrenta ninguna amenaza y no conoce cuáles son las acciones que se deberían tomar para su conservación. Con respecto a si usa o no el agua de lluvia, un 71,4\% aseguró que si para sus actividades domésticas $(90,0 \%)$ y para su ganado $(10,0 \%)$.

En este sentido, el $50,0 \%$ de la población estaría dispuesta a implementar un sistema de colecta de agua de lluvia en sus domicilios.

Solo el 42,9\% realizó un pago por el servicio del agua que oscila entre 2 y 4 soles por mes $(66,7 \%)$. Respecto a cuánto más pagarían por la conservación de la fuente de agua el 78,6\% no haría un pago adicional. Sin embargo, un 14,3\% si pagaría hasta 4 soles más.

Techos de las viviendas y principales amenazas de las fuentes de agua

Se observó que los materiales predominantes de los techos de las viviendas son la calamina, el esternil y la teja (Figura 3a). Con respecto a la principal amenaza que enfrenta la fuente de agua se observó que es la ganadería (Figura 3b). 


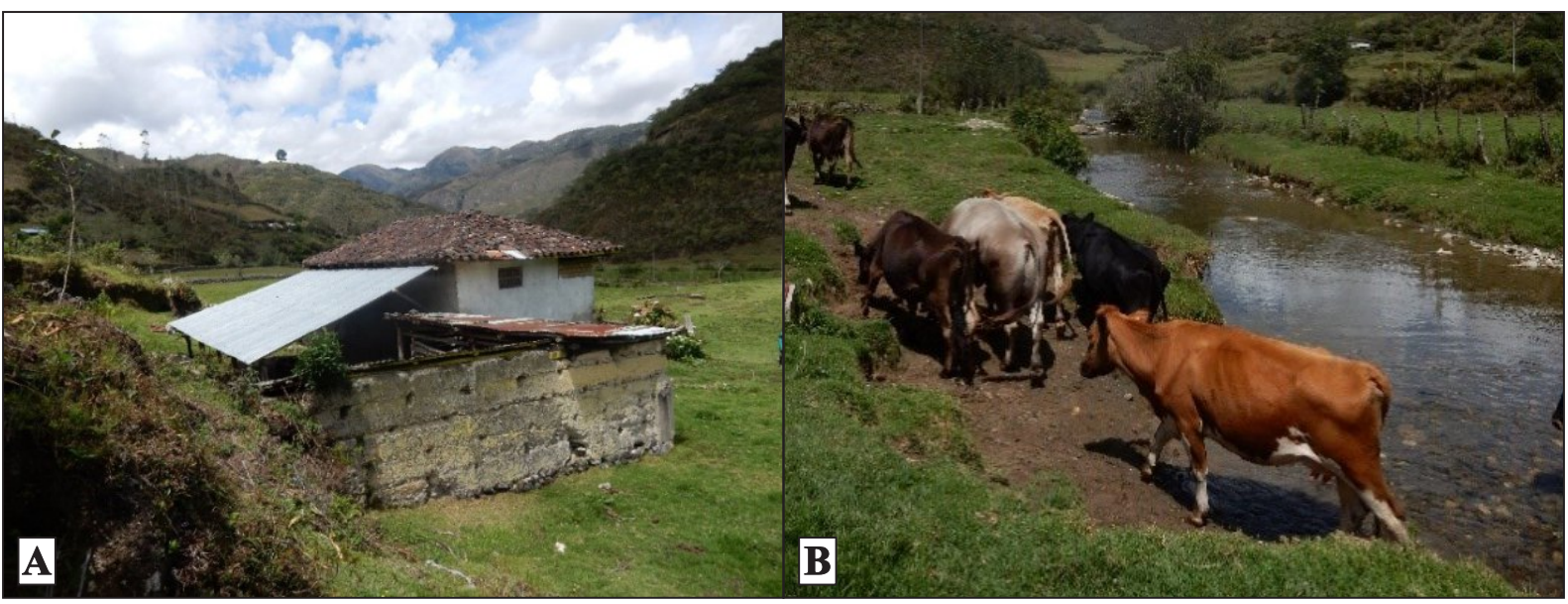

Figura 3. A, Techo de vivienda característico de la zona. B, Principal amenaza a la fuente del agua.

\section{DISCUSIÓN}

El crecimiento poblacional ha generado que la cantidad de desechos producidos también se incremente, lo que aunado a una falta de cultura ambiental repercute en la calidad de recursos naturales como el agua, ya que son los principales depósitos de los residuos generados producto de las diferentes actividades humanas como la actividad agropecuaria y las domésticas (Banco Mundial, 2017).

La principal amenaza a la fuente de agua del anexo de Pomacochas es la actividad ganadera. Esto tiene mucho sentido dado que la actividad pecuaria es la principal actividad del distrito seguido de la agricultura (INEI, 2016). Esto se deja notar en los alrededores de la fuente de agua ya que se observan grandes áreas de pasturas para alimentación de ganado, que en su gran mayoría se conducen con un pastoreo a campo abierto o estaqueo, repercutiendo de manera negativa sobre el suelo y sobre el recurso hídrico.

El material predominante de las viviendas fue el adobe, y los techos de calamina, cuyo material es el más apropiado para realizar la colecta de agua de lluvia, debido a su facilidad de limpieza y a la durabilidad del mismo (Gualdrón, 2014).

Se observó que una gran parte de la población del anexo de Pomacochas tiene disposición a implementar un sistema de cosecha de agua de lluvia en su domicilio, pese a que no sufren de serios problemas de escasez, lo que demuestra que sí tienen interés en proteger la fuente de agua y garantizar su conservación. Sin embargo, un tema de preocupación es que la mayoría de los pobladores no asume la ganadería como una amenaza de la fuente de agua y no muestra su compromiso por emprender acciones para corregir estos problemas, lo que quedaría explicado por la escasa instrucción que poseen ya que la mayoría solo alcanzó la primaria completa (Moreira, 2014).

Un aspecto importante a considerarse en el futuro es que el éxito del proyecto de implementar un sistema de cosecha de agua también dependerá de un correcto diseño, que permita evitar la pérdida de agua por evaporación de la superficie de área expuesta y la pérdida por infiltración en el suelo y los taludes. Además, se debe considerar el uso de materiales apropiados que minimicen o supriman en su totalidad la infiltración, que sean duraderos y que tengan una relación beneficio-costo positiva (Basán, 2011).

Otro aspecto a tener en cuenta es la inclusión social en el acceso a las tecnologías de conservación de los recursos hídricos, ya que de este factor depende que esta tecnología sea sustentable, ayudando a revertir el proceso de degradación ambiental de los recursos hídricos y mejorando el nivel de vida, especialmente de las poblaciones rurales (Lapsenson, 2011).

\section{CONCLUSIONES}

La fuente de agua que abastece el anexo de Pomacochas viene siendo afectada principalmente por la actividad pecuaria y la disposición final inadecuada de los residuos sólidos. Un tema de preocupación es que la 
población practica la actividad ganadera casi en su totalidad. Sin embargo, no la realizan de una manera sustentable, ya que la gran mayoría lon hace a campo abierto y no incluyen el componente arbóreo en sus sistemas de producción, originando una contaminación en la fuente de agua. No obstante, la población no reconoce este tipo de contaminación por lo que no pretende implementar ninguna actividad o acción para solucionar este problemática.

Un aspecto positivo es que al menos la mitad de la población si está dispuesta a implementar un sistema de colecta de agua de lluvia y casi la totalidad de esta tiene un material de sus techos apropiado para la implementación de este sistema. Lo que sería necesario en un futuro para el éxito de la implementación de esta tecnología sería la búsqueda de presupuesto y el cuidado que se le ponga en el diseño, construcción y mantenimiento de los mismos, además de la participación activa de la población que garantice la sostenibilidad de esta tecnología y la sostenibilidad del recurso. Un aspecto importante sería mejorar los sistemas de producción ganadera, para hacerlos más sostenibles. Una manera de conseguirlo sería incluyendo el componente arbóreo.

\section{REFERENCIAS BIBLIOGRÁFICAS}

Abu, M. y A. Tamini. 2011. "Field evaluation of sandditch water harvesting technique in Jordan." Agricultural Water Management 98: 12911296.

ANA (Autoridad Nacional del Agua). 2013. Plan Nacional de Recursos Hídricos. Lima (Perú): ICONO Perú S.A.C.

An, K., Y. Fat, S. Hao, T. Eniolu \& H. Furumai. 2015. "Multi-purpose rainwater harvesting for wáter resource recovery and the coolling effect." Water Research 30: 1-6.

Banco Mundial. 2017. “Crecimiento de la población (\% anual)." https://datos.bancomundial .org/indicador/SP.POP.GROW.

Basán, M. H. 2011. "Sistemas que utilizan agua de lluvia en ambientes rurales." En XXIII Con- greso Nacional del Agua. Resistencia, (Argentina).

Correa, F., J. Urruta, y R. Figueroa. 2011. "Estado del conocimiento y principales amenazas de los humedales boscosos de agua dulce de Chile." Revista Chilena de Historia Natural 84: 325 340.

Delgado, L. E., M. Torres, A. Tironi, y V. Hernán. 2015. "Estrategia de adaptación local al cambio climático para el acceso equitativo al agua en zonas rurales de Chile." América Latina Hoy 69: 113-137.

Gualdrón, N. 2014. Captación de agua lluvia como alternativa comunitaria ante la escasez y la contaminación para el consumo humano y actividades agropecuarias en las veredas el Salado y la Aguada del municipio de Lebrija Santander, Salado y La Aguada del municipio de Lebrija Santander, Colombia. Tesis de Maestría. Universidad de Manizales. Manizales (Colombia).

INEI (Instituto Nacional de Estadística e Informática). 2007. Censos Nacionales 2007 XI de población y VI de vivienda. Sistema de Consulta de Datos de Centros Poblados (CCPP) y Población Dispersa. Lima (Perú): INEI.

INEI (Instituto Nacional de Estadística e Informática). 2016. Perú Síntesis Estadística 2016. Lima (Perú): INEI.

Lapsenson, M. (2011). “Agua e inclusión social en cuencas de la región metropolitana de Buenos Aires." En XXIII Congreso Nacional del Agua. Resistencia, (Argentina).

MINAGRI (Ministerio de Agricultura y Riego). 2012. Política y Estrategia Nacional de Recursos Hídricos. Lima (Perú): MINAGRI.

Moreira, C., F. Araya, y C. Charpentier. 2014. "Educación ambiental para la conservación del recurso hídrico a partir del análisis estadístico de sus variables." Tecnología en Marcha 28 (3): $74-85$.

ONU (Organización de las Naciones Unidas). 2015. 
"Una población en crecimiento" Población. http://www.un.org/es/sections/issuesdepth/population/index.html. (04-07-2017).

Ospina, O., y Y, Moyano. 2015. “Evaluación del aprovechamiento para consumo humano del agua de lluvia en una microcuenca urbana de Ibagué, Tolima, Colombia.” Ingenium 24 (9): 1122

Rashidi, M. H., B. Saghafian, y F. Haghighi. 2013. "Assesment of residential rainwater harvesting efficiency for meeting non-potable wáter demands in three climate conditions." Resources, Conservation and Recycling 73: 86-93.

Ricardo, M. P., M. Méndez, C. B. Pérez, L. O. Sierra, y V. Cutie. 2015. Evaluación de la influencia de la captación de agua de lluvia en casas de cultivos. Revista Ingeniería Agrícola 5 (4): 3 9.

Teodoro, J., R. Moncayo, S. Ochoa, F. Estrada, G. Cruz Cárdena, C. Escalera, F. Villalpando, J. Nava, A. Ramos, y M. López. 2013. "Calidad química del agua subterránea y superficial en la cuenca del río Duero, Michoacán.” Tecnología y Ciencias del Agua 4: 127-146.

UNESCO. 2003. Agua para todos agua para la vida. Informe de las naciones unidas sobre el desarrollo de los recursos hídricos en el mundo. Génova (Suiza): UNESCO.

UNESCO. (2017). Hechos y cifras: la demografia y el consumo son los principales responsables de la presión ejercida sobre los recursos hídricos. Génova (Suiza): UNESCO. 